TITLE:

\title{
Distributed detection of MIMO spatial multiplexed signals in terminal collaborated reception
}

\section{AUTHOR(S):}

Du, Fengning; Murata, Hidekazu; Kasai, Mampei; Nakahira, Toshiro; Ishihara, Koichi; Sasaki, Motoharu; Moriyama, Takatsune

\section{CITATION:}

Du, Fengning ...[et al]. Distributed detection of MIMO spatial multiplexed signals in terminal collaborated reception. IEICE Transactions on Communications 2020, E104-B(7): 884-892

\section{ISSUE DATE:}

2020-07

URL:

http://hdl.handle.net/2433/264522

\section{RIGHT:}

(C) 2020 The Institute of Electronics, Information and Communication Engineers 


\title{
PAPER \\ Distributed Detection of MIMO Spatial Multiplexed Signals in Terminal Collaborated Reception
}

\author{
Fengning DU ${ }^{\dagger}$, Nonmember, Hidekazu MURATA ${ }^{\dagger a}$, Senior Member, Mampei KASAI ${ }^{\dagger}$, Student Member, \\ Toshiro NAKAHIRA ${ }^{\dagger \dagger}$, Koichi ISHIHARA ${ }^{\dagger \dagger}$, Motoharu SASAKI ${ }^{\dagger \dagger}$, Members, \\ and Takatsune MORIYAMA ${ }^{\dagger \dagger}$, Nonmember
}

\begin{abstract}
SUMMARY Distributed detection techniques of multiple-input multiple-output (MIMO) spatially multiplexed signals are studied in this paper. This system considered employs multiple mobile stations (MSs) to receive signals from a base station, and then share their received signal waveforms with collaborating MSs. In order to reduce the amount of traffic over the collaborating wireless links, distributed detection techniques are proposed, in which multiple MSs are in charge of detection by making use of both the shared signal waveforms and its own received waveform. Selection combining schemes of detected bit sequences are studied to finalize the decisions. Residual error coefficients in iterative MIMO equalization and detection are utilized in this selection. The error-ratio performance is elucidated not only by computer simulations, but also by offline processing using experimental signals recorded in a measurement campaign.

key words: multi-user MIMO, transmission experiment, interference cancellation, terminal collaboration, collaborative reception, measurement campaign
\end{abstract}

\section{Introduction}

Mobile terminal collaboration is a new type of reception technique. In [1], [2], this concept was studied for broadcasting channels. In [3]-[6], this concept was also considered for a multiple-input multiple-output (MIMO) scenario to increase capacity of a radio channel by using multiple transmit and receive antennas. This system employs multiple mobile stations (MSs) to receive signals from a base station (BS), and then share their received signals with collaborating MSs through dedicated inter-MS wireless links that are referred to as collaboration links hereafter.

A conventional multiple user MIMO system requires instantaneous channel state information (CSI) at the BS for precoding. In mobile channels, CSI gets outdated quickly over time. The collaborated MIMO reception does not need instantaneous CSI feedback for precoding. Therefore, it is suitable for moving MSs [7] as an alternative to the MIMO systems with precoding. Particularly, in public transportation environments such as buses and trains, the collaborating MSs are close to each other and there is almost no change in relative positions during their movement.

Manuscript received July 16, 2020.

Manuscript revised November 9, 2020.

Manuscript publicized December 29, 2020.

$\dagger$ The authors are with the Graduate School of Informatics, Kyoto University, Kyoto-shi, 606-8501 Japan.

${ }^{\dagger}$ The authors are with NTT Access Network Service Systems Laboratories, NTT Corporation, Yokosuka-shi, 239-0847 Japan.

a)E-mail: murata@i.kyoto-u.ac.jp

DOI: 10.1587/transcom.2020EBP3108
A key issue to realize a collaborated MIMO reception system is the throughput and latency performance of collaboration links. This issue always has been crucial for cooperative/collaborative wireless networks. We can expect to leverage recently-developed high-throughput and lowlatency transmission techniques in higher frequency bands.

In collaborated MIMO reception systems, the more collaboration MSs we have, the better reception performance can be expected [8]. However, it is better to choose a small subset of collaboration MSs in order to reduce the amount of both power consumption and traffic over the collaboration links [9]-[13]. In [9], [10], [12], [13], channel matrices based MS selection schemes were investigated. However, the perfect selection scheme (i.e. genie-aided selection) performed considerably better.

In this paper, we propose a distributed detection scheme in order not only to reduce the amount of traffic over the collaboration links but also to improve the error-ratio performance of collaborated MIMO reception. This scheme employs residual error coefficients as a metric for selection combining of detected bit sequences at the expense of additional computational complexity. The error-ratio performance is revealed via computer simulations and offline processing of received signal waveforms in an actual environment.

\section{System Descriptions}

\subsection{System Model}

A BS transmits $M$ spatially-multiplexed independent signal streams to $N$ MSs on the same carrier frequency at the same time. At the receiver side, each MS equipped with a single antenna shares the received signal with other MSs through collaboration links. Thus, the signals received by MSs are utilized for detection in a collaborative manner.

Let $\mathcal{N}(|\mathcal{N}|=N)$ be a set of all MSs involved in terminal collaborated MIMO reception, and a subset $\mathcal{L} \subset \mathcal{N}$ be a set of selected MSs for detection. In other words, $L=|\mathcal{L}|$ received signals from the BS are used for MIMO detection.

Figure 1 shows a general system model of terminal collaborated reception. At each detection MS, MIMO detection is carried out. Then, a master MS finalizes the decisions based on the detected bit sequences and associated information from the detection MSs. The finalized bit sequences 


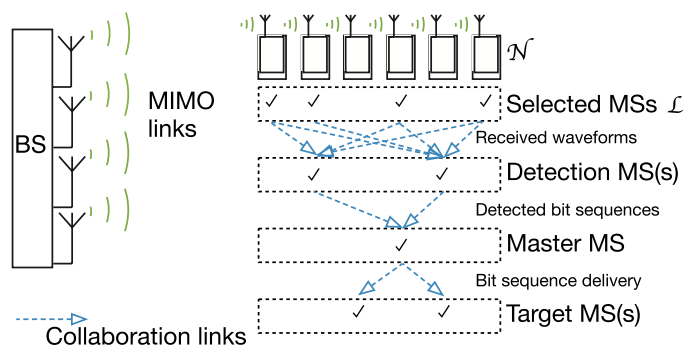

Fig. 1 General system model of terminal collaborated reception.

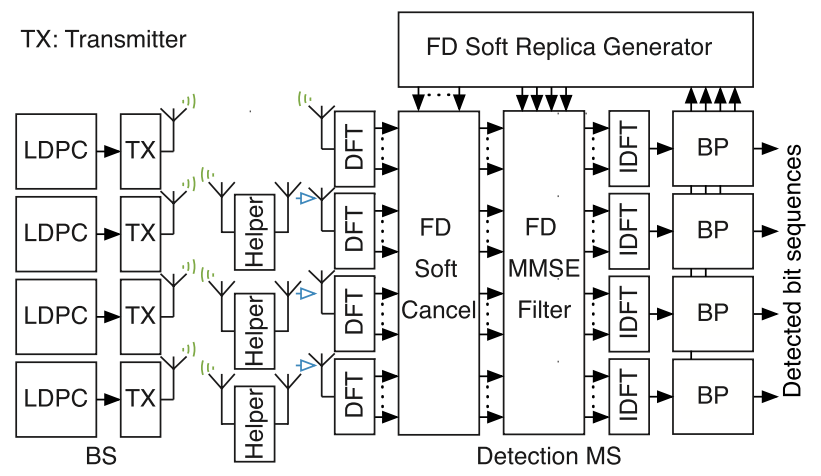

Fig. 2 Signal transmission and reception model $(M=L=4)$.

are delivered to target MSs. The amount of traffic over the collaboration links greatly depends on the selection of these MSs. We assume that one of the detection MSs also serve as the master MS and the target MS for simplicity.

2.2 Frequency-Domain Iterative Equalization and Detection

A signal transmission and reception model is shown in Fig. 2. This figure shows the case that the detection MS is included in $\mathcal{L}$. Thus, $L-1 \mathrm{MSs}$ act as relay (helper) MSs for this detection MS. A single carrier frequency-domain soft-cancel/minimum mean square error (MMSE) iterative equalizer [14], [15] is employed. This equalizer combines three processes: i) MMSE frequency-domain (FD) equalization, ii) soft decoding of a low-density parity-check (LDPC) code by belief propagation (BP), and iii) soft cancellation.

Let $\boldsymbol{y}_{\mathcal{L}}(k)$ be $\left[y_{1}(k), y_{2}(k), \ldots, y_{L}(k)\right]^{\mathrm{T}} \in \mathbb{C}^{L \times 1}$ where $y_{l}(k)$ is the received signal at the $k$ th $(1 \leq k \leq K)$ symbol of the $l$ th MS in $\mathcal{L}$, and the superscript $(.)^{\mathrm{T}}$ denotes the transpose operation. The FD received signals $\boldsymbol{y}_{\mathcal{L}}(f) \in \mathbb{C}^{L \times 1}$ are equalized by MMSE filters. The equalized signals $\tilde{\boldsymbol{x}}(f) \in \mathbb{C}^{M \times 1}$ are converted to the signals $\tilde{\boldsymbol{x}}(k) \in \mathbb{C}^{M \times 1}$ in the time-domain. Then, BP calculates log likelihood ratios (LLRs) $L\left(c_{m, k, i}\right)$ during inner iterations, where $c_{m, k, i}$ is the $i$ th bit of the $k$ th symbol of the $m$ th transmitted stream. In an FD soft replica generator, soft-decision symbols $\hat{\boldsymbol{x}}(k)=\left[\hat{x}_{1}(k), \hat{x}_{2}(k), \ldots, \hat{x}_{M}(k)\right]^{\mathrm{T}} \in \mathbb{C}^{M \times 1}$ are generated as follows in the case of quadrature phase-shift keying (QPSK) modulation:

$$
\begin{aligned}
\hat{x}_{m}(k)= & \frac{1}{\sqrt{2}} \tanh \left(L\left(c_{m, k, 1}\right) / 2\right) \\
& +\frac{1}{\sqrt{2}} \tanh \left(L\left(c_{m, k, 2}\right) / 2\right) \sqrt{-1} .
\end{aligned}
$$

The symbols $\hat{\boldsymbol{x}}(k)$ are converted to the signals $\hat{\boldsymbol{x}}(f)=$ $\left[\hat{x}_{1}(f), \hat{x}_{2}(f), \ldots, \hat{x}_{M}(f)\right]^{\mathrm{T}} \in \mathbb{C}^{M \times 1}$ in the FD. Next, softdecision replicas $\hat{\boldsymbol{y}}_{\mathcal{L}, m}(f) \in \mathbb{C}^{L \times 1}$ are generated as follows:

$$
\hat{\boldsymbol{y}}_{\mathcal{L}, m}(f)=\boldsymbol{h}_{\mathcal{L}, m}(f) \hat{x}_{m}(f),
$$

where $\boldsymbol{h}_{\mathcal{L}, m}(f) \in \mathbb{C}^{L \times 1}$ is the $m$ th column of the estimated channel matrix $\boldsymbol{H}_{\mathcal{L}}(f)$ in the FD. Equalized signals by MMSE filters with soft-cancellation can be expressed as

$$
\begin{aligned}
\tilde{x}_{m}(f)=\boldsymbol{w}_{\mathcal{L}, m}^{\mathrm{H}}(f) & \left\{\boldsymbol{y}_{\mathcal{L}}(f)-\sum_{i \neq m} \hat{\boldsymbol{y}}_{\mathcal{L}, i}(f)\right\}, \\
\boldsymbol{w}_{\mathcal{L}, m}(f)= & \left(\boldsymbol{h}_{\mathcal{L}, m}(f) \boldsymbol{h}_{\mathcal{L}, m}^{\mathrm{H}}(f)\right. \\
& \left.+\sum_{i \neq m} \beta_{\mathcal{L}, i} \boldsymbol{h}_{\mathcal{L}, i}(f) \boldsymbol{h}_{\mathcal{L}, i}^{\mathrm{H}}(f)+\sigma^{2} \mathbf{I}_{L}\right)^{-1} \boldsymbol{h}_{\mathcal{L}, m}(f),
\end{aligned}
$$

where $\sigma^{2}$ is the noise variance, $\mathbf{I}_{L}$ is the $L \times L$ identity matrix, the superscript $(.)^{\mathrm{H}}$ represents the Hermitian transpose of a matrix, and $\boldsymbol{w}_{\mathcal{L}, m}(f) \in \mathbb{C}^{L \times 1}$ is an MMSE filter with residual error coefficients $\beta_{\mathcal{L}, m}\left(0 \leq \beta_{\mathcal{L}, m} \leq 1\right)$ shown below [14], [16]

$$
\beta_{\mathcal{L}, m}= \begin{cases}0, & \text { Parity-check satisfied } \\ 1-\frac{1}{K} \sum_{k}\left|\hat{x}_{m}(k)\right|^{2}, & \text { otherwise. }\end{cases}
$$

As shown in Eq. (5), if all the parity-check equations of the $m$ th stream are satisfied for hard decisions formed on the a posteriori LLRs, let $\beta_{\mathcal{L}, m}$ be 0 . The coefficients $\beta_{\mathcal{L}, m}$ represent the average residual symbol interference after cancellation [16].

These processes, namely Eqs. (1)-(5) and soft decoding, are repeated up to a predetermined number of times as an outer iteration.

\subsection{Early Stopping}

Early stopping (ES) is employed for experimental performance evaluation [17]. The residual error coefficients shown in Eq. (5) are utilized as a stopping metric of ES. After calculating Eq. (5), the outer iteration is terminated if the following inequality holds:

$$
\sum_{m=1}^{M} \beta_{\mathcal{L}, m} \leq \varepsilon .
$$

By adjusting the iteration control threshold $\varepsilon$, the number of outer iterations can be reduced. Note that ES improves the error-ratio performance of experiments [17]. However, it offers almost no improvement in the error-ratio performance 
of computer simulations assuming simple propagation channels. Further investigation clarifies the reason behind this observation.

\subsection{Residual Error Coefficients Based MS Selection}

Residual error coefficients based MS selection schemes are described as follows [18]. In a full search scheme (Full), the best subset of MSs among all of the possible subsets of MSs is selected in terms of the sum of $\beta_{\mathcal{L}, m}$ of the last outer iteration as follows:

$$
\mathcal{L}_{\text {Full }}^{*}=\underset{\substack{\mathcal{L} \subset \mathcal{N} \\|\mathcal{L}|=L}}{\arg \min } \sum_{m} \beta_{\mathcal{L}, m .}
$$

The selected subset of MSs $\mathcal{L}_{\text {Full }}^{*}$ is used for detection of all streams. In this scheme, detection process is carried out for all of the possible subsets of MSs. Therefore, at least $N-1$ received waveforms are transmitted over the collaboration links.

In a full search per stream scheme (FullPS), the best subset of MSs is selected for each stream independently as follows:

$$
\mathcal{L}_{\text {FullPS, } m}^{*}=\underset{\substack{\mathcal{L} \subset \mathcal{N} \\|\mathcal{L}|=L}}{\arg \min } \beta_{\mathcal{L}, m} .
$$

These two schemes, namely Full and FullPS, give the performance in the case of whole search space. In these two schemes, the amount of collaboration traffic and the signal processing complexity are major concerns. Moreover, advantage over $N$ MSs collaboration needs to be clarified.

\subsection{Distributed Detection and Combining}

Distributed detection and combining schemes are proposed in order to reduce the number of transmitted waveforms over the collaboration links while still enabling MS subset selection. As shown in Fig. 3 , all MSs in a subset $\mathcal{N}_{1}$ transmit their received waveforms over the collaboration links. All MSs in another subset $\mathcal{N}_{2}$ receive these waveforms, and detect bit sequences of the transmitted streams from the BS using the received waveforms together with its own received waveform from the BS.

This scheme can be described in the same way as Full. In a $d$-detector scheme ( $d$ Det), the best subset of MSs is

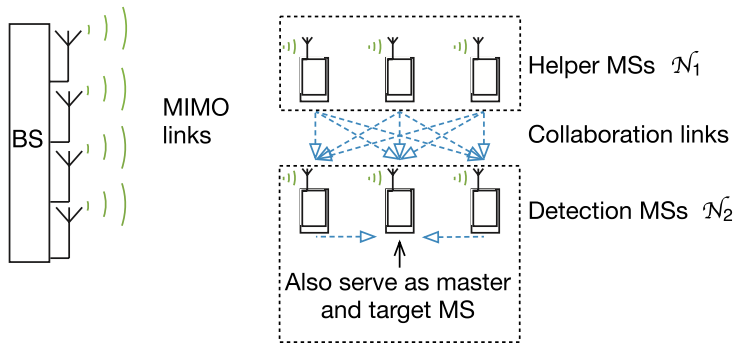

Fig. 3 Distributed MIMO detection and combining. selected as follows:

$$
\mathcal{L}_{d \text { Det }}^{*}=\underset{\substack{\mathcal{L}=\mathcal{N}_{1} \bigcup\{n\} \\ n \in \mathcal{N}_{2} \\|\mathcal{L}|=L}}{\arg \min } \sum_{m} \beta_{\mathcal{L}, m},
$$

where $\mathcal{N}_{1}$ includes $N-d$ MSs as helper MSs, and $\mathcal{N}_{2}=$ $\mathcal{N} \backslash \mathcal{N}_{1}$ includes $d$ MSs as detection MSs.

In a $d$-detector per stream scheme ( $d$ DetPS), the best subset of MSs is selected for each stream independently as shown below:

$$
\mathcal{L}_{d \operatorname{DetPS}, m}^{*}=\underset{\substack{\mathcal{L}=\mathcal{N}_{1} \cup\{n\} \\ n \in \mathcal{N}_{2} \\|\mathcal{L}|=L}}{\arg \min } \beta_{\mathcal{L}, m} .
$$

In these distributed detection and combining schemes, the $N-d$ helper MSs transmit their received waveforms from the BS, and then the $d$ detection MSs carry out detection process by making use of the $L=N-d+1$ received signals from the BS. In total, $d$ candidates of detected bit sequences become available. The master MS selects the best bit sequence for each stream according to Eq. (9) or Eq. (10). This process imposes extra traffic over the collaboration links. However, the amount of traffic to be transmitted for the detected bit sequence gathering is generally less than that of the received waveforms though this issue is depending on implementation details. It is reasonable to include the master and the target MSs in $\mathcal{N}_{2}$.

\subsection{Channel Matrices Based MS Selection Schemes}

Three MS selection schemes based on channel matrices are considered for comparison purpose. In these schemes, training sequences (or estimated CSI) are shared first, then MSs are selected, and finally the remaining parts of the packets are shared for detection [9]. By the singular value decomposition theorem, the estimated channel matrices $\boldsymbol{H}_{\mathcal{L}}^{(f)} \in \mathbb{C}^{L \times M}$ at the frequency index $f$ can be written as:

$$
\boldsymbol{H}_{\mathcal{L}}^{(f)}=\boldsymbol{U}_{\mathcal{L}}^{(f)} \boldsymbol{\Sigma}_{\mathcal{L}}^{(f)} \boldsymbol{V}_{\mathcal{L}}^{(f) \mathrm{H}}
$$

where $\boldsymbol{U}_{\mathcal{L}}^{(f)} \in \mathbb{C}^{L \times L}$ and $\boldsymbol{V}_{\mathcal{L}}^{(f)} \in \mathbb{C}^{M \times M}$ are unitary matrices, and $\Sigma_{\mathcal{L}}^{(f)} \in \mathbb{C}^{L \times M}$ has non-negative diagonal entries. Singular values are the diagonal entries of $\Sigma_{\mathcal{L}}^{(f)}$, and square roots of eigenvalues $\lambda_{\mathcal{L}, i}^{(f)}$ from $\boldsymbol{H}_{\mathcal{L}}^{(f)} \boldsymbol{H}_{\mathcal{L}}^{(f) \mathrm{H}}$ or $\boldsymbol{H}_{\mathcal{L}}^{(f) \mathrm{H}} \boldsymbol{H}_{\mathcal{L}}^{(f)}$, where $i$ is the index of the eigenvalues.

In a maximum product of singular values MS selection scheme (MPoSV) [10], [12], L MSs are selected frame by frame based on these singular values. This scheme selects an MS subset $\mathcal{L}_{\mathrm{MPoSV}}^{*}$ that maximizes the product of all the singular values as shown below:

$$
\mathcal{L}_{\mathrm{MPoSV}}^{*}=\underset{\substack{\mathcal{L} \subset \mathcal{N} \\|\mathcal{L}|=L}}{\arg \max } \prod_{i, f} \sqrt{\lambda_{\mathcal{L}, i}^{(f)}}
$$

In a minimum sum of condition numbers scheme 
(MCN) [12], [19], the MS subset $\mathcal{L}_{\mathrm{MCN}}^{*}$ that minimizes the sum of condition numbers is selected as shown below:

$$
\mathcal{L}_{\mathrm{MCN}}^{*}=\underset{\substack{\mathcal{L} \subset \mathcal{N} \\|\mathcal{L}|=L}}{\arg \min } \sum_{f} c n_{\mathcal{L}}^{(f)},
$$

where

$$
c n_{\mathcal{L}}^{(f)}=\frac{\max _{i} \sqrt{\lambda_{\mathcal{L}, i}^{(f)}}}{\min _{i} \sqrt{\lambda_{\mathcal{L}, i}^{(f)}}} .
$$

In a signal to interference plus noise ratio per stream scheme (SINRPS) [9], [13], the sum of estimated SINRs is maximized for each stream independently as shown below:

$$
\mathcal{L}_{\text {SINRPS }, m}^{*}=\arg \max _{\substack{\mathcal{L} \subset \mathcal{N} \\|\mathcal{L}|=L}} \sum_{f} \gamma_{\mathcal{L}, m}^{(f)}
$$

where $\gamma_{\mathcal{L}, m}^{(f)}$ is an estimated SINR of the $m$ th stream for $\mathcal{L}$ assuming equal transmit power for each stream given as [20]

$$
\gamma_{\mathcal{L}, m}^{(f)}=\boldsymbol{h}_{\mathcal{L}, m}^{(f) \mathrm{H}}\left(\sum_{l=1, l \neq m}^{M} \boldsymbol{h}_{\mathcal{L}, l}^{(f)} \boldsymbol{h}_{\mathcal{L}, l}^{(f) \mathrm{H}}+\sigma^{2} \mathbf{I}_{L}\right)^{-1} \boldsymbol{h}_{\mathcal{L}, m}^{(f)} .
$$

The vector $\boldsymbol{h}_{\mathcal{L}, m}^{(f)}$ is the $m$ th column of $\boldsymbol{H}_{\mathcal{L}}^{(f)}$. Note that SINRPS may require more collaboration traffic since this scheme may select a different subset of MSs for each stream.

\section{Computer Simulations}

Computer simulations were carried out to evaluate the frame error-ratio (FER) performance of Full, FullPS, 3Det, 3DetPS, MPoSV, MCN, and SINRPS assuming frequencyflat and frequency-selective fading channels. In addition, a fixed terminal selection scheme (Fixed) is also considered for comparison purpose. In Fixed, the subset remained fixed during the entire FER measurement period. Each packet included one of 39-symbol orthogonal training sequences (TS), a control sequence (CTRL), a 4-symbol cyclic prefix $(\mathrm{CP})^{\dagger}$, and a 192-symbol data section (DATA) as shown later in Sect. 4. The data section was rate 1/2 LDPC encoded, and modulated by QPSK modulation. The data bits were successively drawn from a pseudo-random sequence. Channel coefficients up to seven symbols delay were estimated by a simple least-square technique. The numbers of inner iterations (i.e. BP) and outer iterations were eight and three, respectively.

Figure 4 shows the FER performance versus the average signal-to-noise ratio (SNR) in a frequency-flat independently and identically distributed (i.i.d.) Rayleigh fading channel with the normalized Doppler frequency $f_{\mathrm{D}} / T_{\mathrm{S}}=6.4 \times 10^{-5}$,

\footnotetext{
${ }^{\dagger}$ Due to minor limitations in the experimental equipment, the length of the cyclic prefix was set to four in both the computer simulations and the experiment.
}

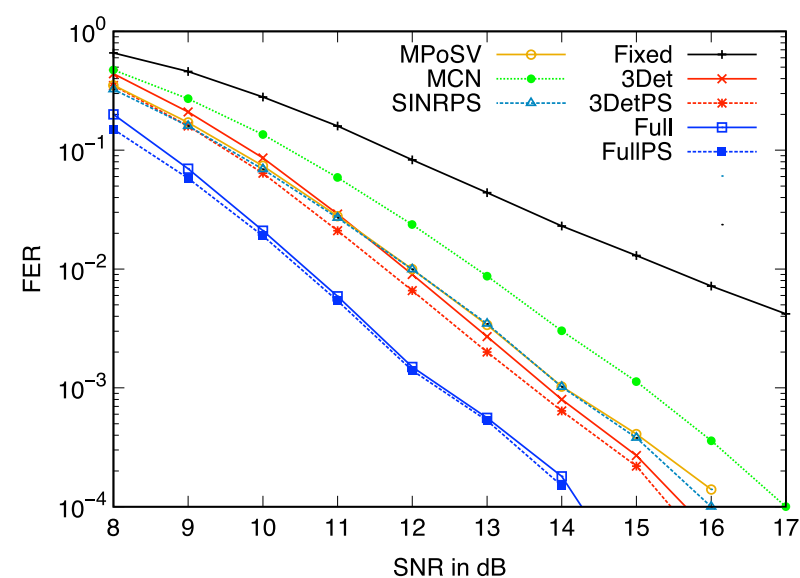

Fig. 4 FER performance obtained by computer simulations assuming frequency-flat Rayleigh fading channel.

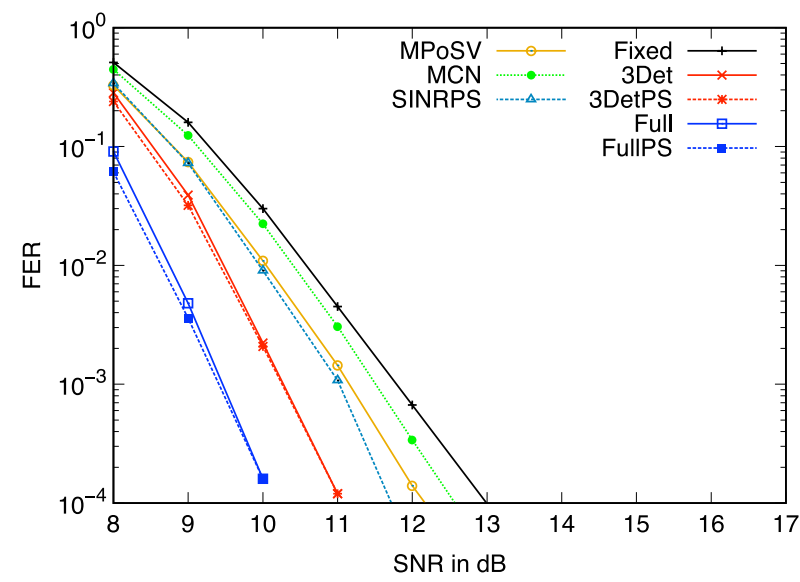

Fig. 5 FER performance obtained by computer simulations assuming 4path symbol-spaced equal-power frequency-selective i.i.d. Rayleigh fading channel.

where $f_{\mathrm{D}}$ is the maximum Doppler frequency, and $T_{\mathrm{s}}$ is the symbol period. The average signal power included all four streams $(M=4)$. The four $(L=4)$ signals out of the six signals $(N=6)$ were used for equalization and detection. As can be seen, 3Det and 3DetPS outperformed both Fixed and MCN. Furthermore, the performance of 3Det and 3DetPS was superior to that of MPoSV and SINRPS in the high SNR region. Full and FullPS performed even better. The FER performance of 3DetPS was around $0.25 \mathrm{~dB}$ better than that of 3Det in contrast to Full and FullPS where both schemes performed almost the same. This indicates that three subsets were insufficient but 15 subsets were enough to find a common subset for detection of four streams.

Figure 5 shows the FER performance versus average SNR in 4-path symbol-spaced equal-power frequencyselective i.i.d. Rayleigh fading channel. The FER performance of all the schemes was improved significantly. In this channel, 3Det and 3DetPS performed almost the same especially in the low FER region. This is because the FER performance is lower bounded by the probability that there is a stream which has errors in all the subsets. 


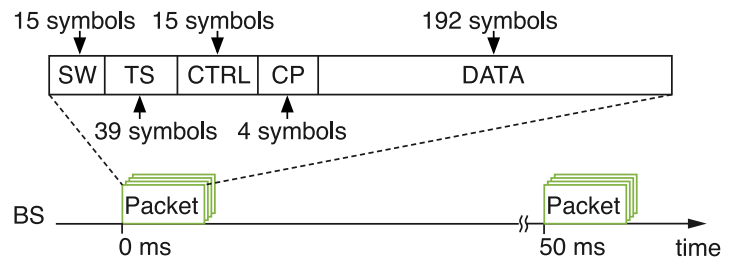

Fig. 6 Frame structure.

Table 1 Major parameters of outdoor measurement campaign.

\begin{tabular}{lr}
\hline Parameters & Values \\
\hline Number of BS antennas & 4 \\
Transmit power of BS & $1 \mathrm{~W}$ \\
Gain of BS antenna & $5.8 \mathrm{dBi}$ \\
Cable loss & $1.4 \mathrm{~dB}$ \\
BS antenna height & $25.5 \mathrm{~m}$ \\
Carrier frequency & $427.2 \mathrm{MHz}$ \\
Symbol rate & $312.5 \mathrm{ksps}$ \\
Transmit filter & Square root raised cosine \\
& Roll-off factor 0.4 \\
Frame interval & $50 \mathrm{~ms}$ \\
\hline Number of MSs & 6 \\
Number of antennas of each MS & 1 \\
Antenna of MS & $\lambda / 4$ omnidirectional monopole \\
MS antenna height & $2.1 \mathrm{~m}$ \\
\hline
\end{tabular}

\section{Field Experiment}

\subsection{Experimental Setup}

The major parameters of an outdoor measurement campaign are shown in Table 1. Four $(M=4)$ packets were transmitted in every $50 \mathrm{~ms}$ frame by using QPSK modulation as shown in Fig. 6. The symbol rate of each packet was 312.5 kilo symbols per second (ksps). The packet consisted of a synchronization sequence (SW), one of orthogonal TS, CTRL, $\mathrm{CP}$, and DATA. As in the computer simulation, the data bits were successively drawn from a pseudo-random sequence. Therefore, the four packets were independently generated, encoded, and modulated frame by frame in real-time.

As shown in Fig. 7, four BS antennas were located $25.5 \mathrm{~m}$ above the ground. These antennas were $5.8 \mathrm{dBi}$ omnidirectional. Signals were transmitted at the carrier frequency $427.2 \mathrm{MHz}$ with the transmit power $1 \mathrm{~W}$ per antenna. As shown in Fig. 8 , six $(N=6)$ MSs' receive antennas were set on a roof of a vehicle ( $2.1 \mathrm{~m}$ height). These antennas were omnidirectional monopole, and arranged as a uniform circular array. The timings and frequencies of the entire system were based on 1-pulse-per-second signals and $10 \mathrm{MHz}$ signals of global positioning system receivers. Therefore, the SW was just used to confirm proper operations.

In this paper, a subset $\mathcal{L}(|\mathcal{L}|=L=4)$ of the six received signals from the BS were selected and used for equalization and detection. An MS subsystem installed in the vehicle has inter-MS collaboration links at $12.9 \mathrm{GHz}$, and can perform all the signal processing tasks of a single subset in real time. In order to examine the FERs of all possible subsets, the received signal waveform from the BS was

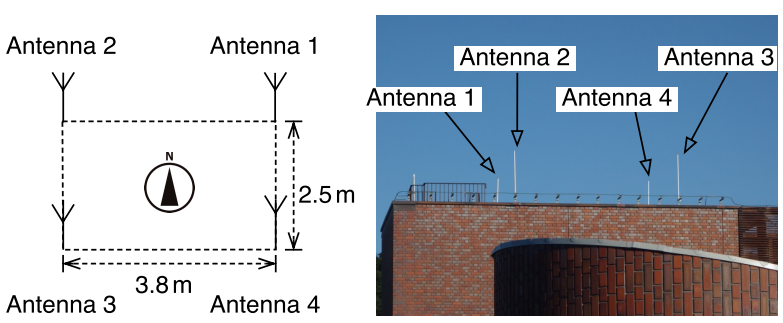

Fig. 7 BS antennas.

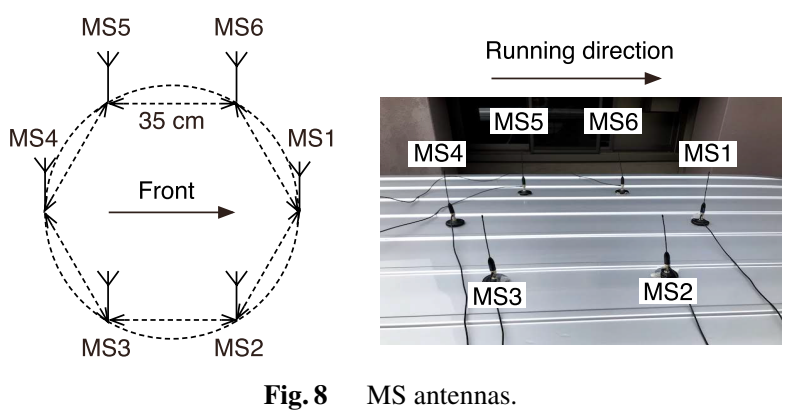

recorded at each MS [12], [17], [18] and used for offline processing. Therefore, there was no inter-MS communication for collaboration in this paper.

The maximum number of outer iterations was three, and the actual number of iterations of each frame was controlled by ES with the iteration control threshold $\varepsilon=0.01$. The noise variance was determined to achieve the best performance.

As shown in Fig. 9, we drove the vehicle on Imadegawadori and Shirakawa-dori streets in Kyoto city to record the actual received signal waveforms. Received power calibration was performed for all MSs before the experiment. We conducted the experiments twice (i.e. trial 1 and 2) to verify the results.

In this experiment, most of the received power were included in the estimated channel coefficients up to threesymbol delay (four taps). Through the use of a 4-point complex discrete Fourier transform, four frequencies were considered for the FD channel matrices.

\subsection{Experimental Results}

The FER performance is shown in Figs. 10 and 11. The FER was averaged over the entire measurement course. Note that absolute FERs values differ between trial 1 and trial 2 due to the different traffic condition (e.g. vehicle speed, lane position, other vehicles). The helper MS group was $\{$ MS1, MS2, MS3 \}, and remained unchanged during the measurement. Therefore, the proposed schemes, 3Det and 3DetPS, detected three bit sequences corresponding to the MS subset indexes $\# 1=\{$ MS1, MS2, MS3, MS4 $\}, \# 2=\{$ MS1, MS2, MS3, MS5 $\}$, and $\# 3=\{$ MS1, MS2, MS3, MS6 $\}$. Table 2 shows the MS subset index and its MSs.

From these figures, it is clearly confirmed that the FER performance of 3Det and 3DetPS is superior to that of Fixed 


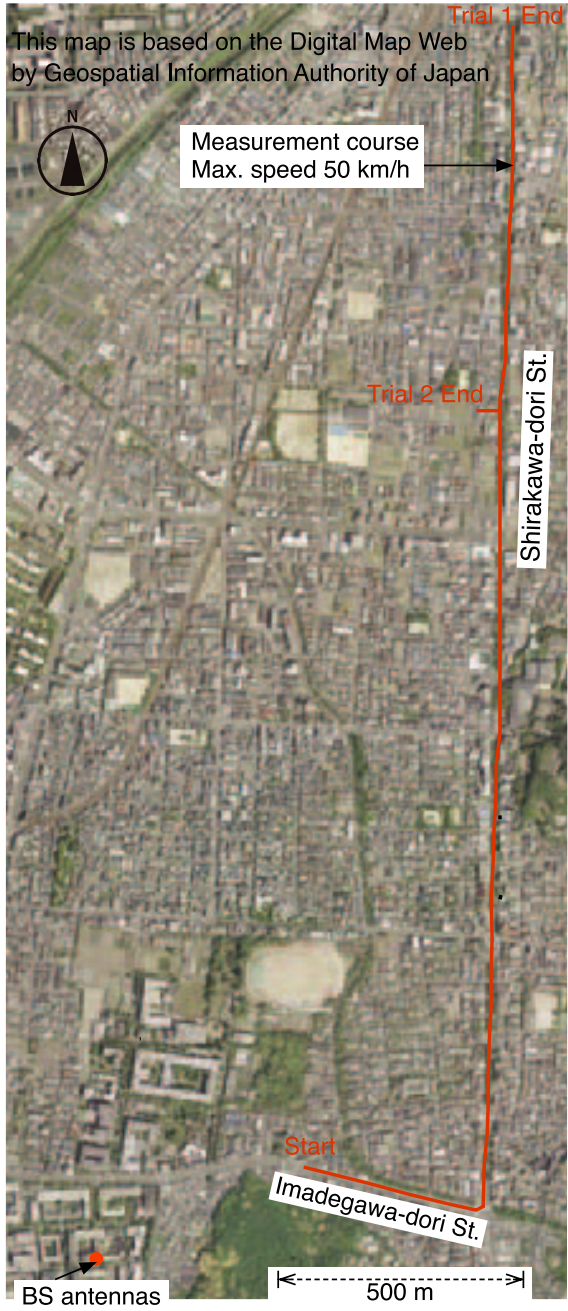

Fig. 9 Measurement campaign course. Course is indicated in red.

Table 2 MS subset index.

\begin{tabular}{|c|c|c|c|}
\hline Index & $\mathcal{L}$ & Index & $\mathcal{L}$ \\
\hline$\# 1$ & $\{\mathrm{MS} 1, \mathrm{MS} 2, \mathrm{MS} 3, \mathrm{MS} 4\}$ & \#9 & $\{$ MS1, MS3, MS5, MS6 $\}$ \\
\hline \#2 & $\{\mathrm{MS} 1, \mathrm{MS} 2, \mathrm{MS} 3, \mathrm{MS} 5\}$ & \#A & $\{\mathrm{MS} 1, \mathrm{MS} 4, \mathrm{MS} 5, \mathrm{MS} 6\}$ \\
\hline \#3 & $\{\mathrm{MS} 1, \mathrm{MS} 2, \mathrm{MS} 3, \mathrm{MS} 6\}$ & \#B & $\{\mathrm{MS} 2, \mathrm{MS} 3, \mathrm{MS} 4, \mathrm{MS} 5\}$ \\
\hline \#4 & $\{\mathrm{MS} 1, \mathrm{MS} 2, \mathrm{MS} 4, \mathrm{MS} 5\}$ & \#C & $\{\mathrm{MS} 2, \mathrm{MS} 3, \mathrm{MS} 4, \mathrm{MS} 6\}$ \\
\hline \#5 & $\{\mathrm{MS} 1, \mathrm{MS} 2, \mathrm{MS} 4, \mathrm{MS} 6\}$ & \#D & $\{\mathrm{MS} 2, \mathrm{MS} 3, \mathrm{MS} 5, \mathrm{MS} 6\}$ \\
\hline \#6 & $\{$ MS1, MS2, MS5, MS6 $\}$ & \#E & $\{\mathrm{MS} 2, \mathrm{MS} 4, \mathrm{MS} 5, \mathrm{MS} 6\}$ \\
\hline \#7 & $\{$ MS1, MS3, MS4, MS5 $\}$ & $\# \mathrm{~F}$ & $\{$ MS3, MS4, MS5, MS6 $\}$ \\
\hline \#8 & $\{\mathrm{MS} 1, \mathrm{MS} 3, \mathrm{MS} 4, \mathrm{MS} 6\}$ & & \\
\hline
\end{tabular}

with any MS subset index. The channel matrices based MS selection schemes, namely MPoSV, MCN, and SINRPS, offered better FER performance than that of Fixed. The proposed two schemes, 3Det and 3DetPS, which are based on the residual error coefficients, achieved even better FER performance. This improvement is due to adaptive selection of detected bit sequences based on the residual error coefficients. As can be seen, 3DetPS offered slightly better FERs than that of 3Det. However, no difference can be found between the FERs of Full and FullPS. FERs of Full and FullPS are greatly smaller than those of 3Det and 3DetPS. This re-

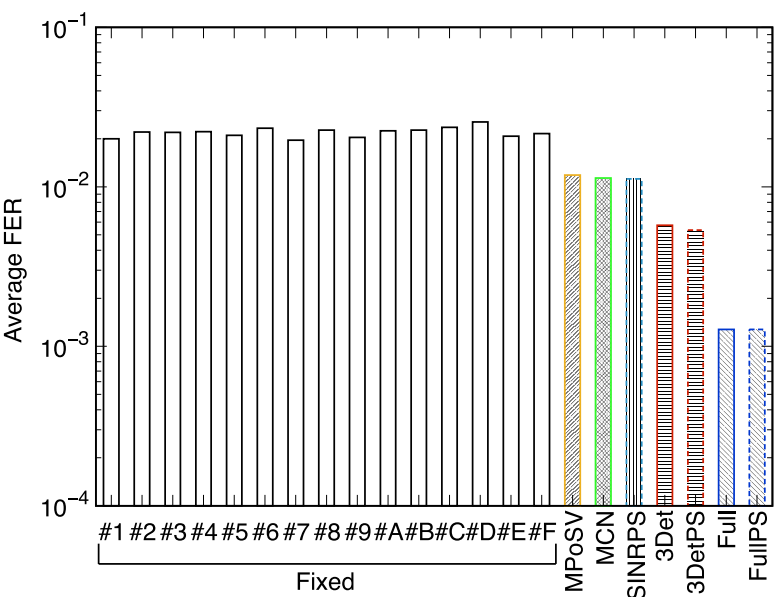

Fig. 10 FER comparisons of trial 1.

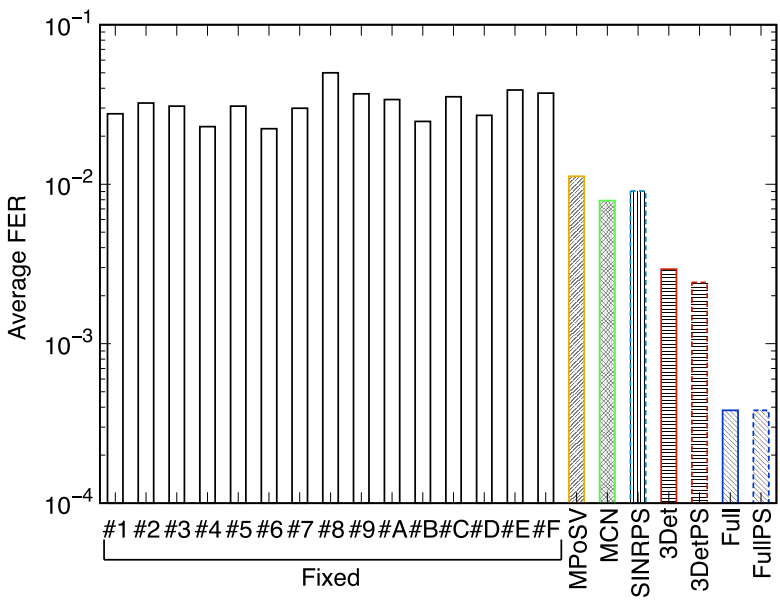

Fig. 11 FER comparisons of trial 2.

sult suggests that the FER performance of 3Det and 3DetPS will be further improved by selecting a helper MS group adaptively. However, this still requires further research.

Figures 12 and 13 show the experimental results versus the frame count of trial 1 and trial 2, respectively. At the top of each figure, received signal strength indicator (RSSI) values averaged over six MSs are shown. In the middle of each figure, the sum of condition number $\sum_{f} c n_{\# 1}^{(f)}$ is also shown. At the bottom of each figure, the FER performance of Fixed \#1, MPoSV, MCN, SINRPS, 3DetPS, and FullPS averaged over 100 frames is shown.

It is difficult to find a clear relationship among RSSI values, sum of condition numbers, and FER performance from these experimental results. However, it is clearly demonstrated that the FER performance of 3DetPS is significantly superior to those of Fixed \#1, MPoSV, MCN, and SINRPS. Moreover, the FERs of FullPS are the same as or better than those of 3DetPS for all 100-frame fragments. This indicates that the residual error coefficient is reliable even in actual propagation conditions.

The numbers of transmitted waveforms in the collaboration links are summarized in Table 3. In Fixed, MPoSV, 


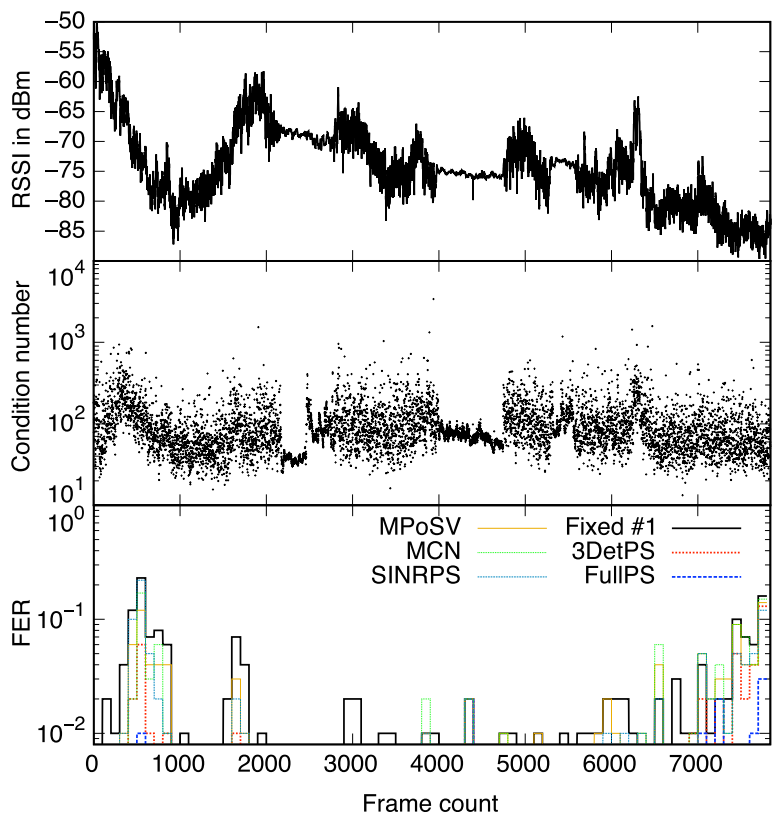

Fig. 12 RSSI, sum of condition numbers, and FER of trial 1. RSSI is averaged over six MSs.

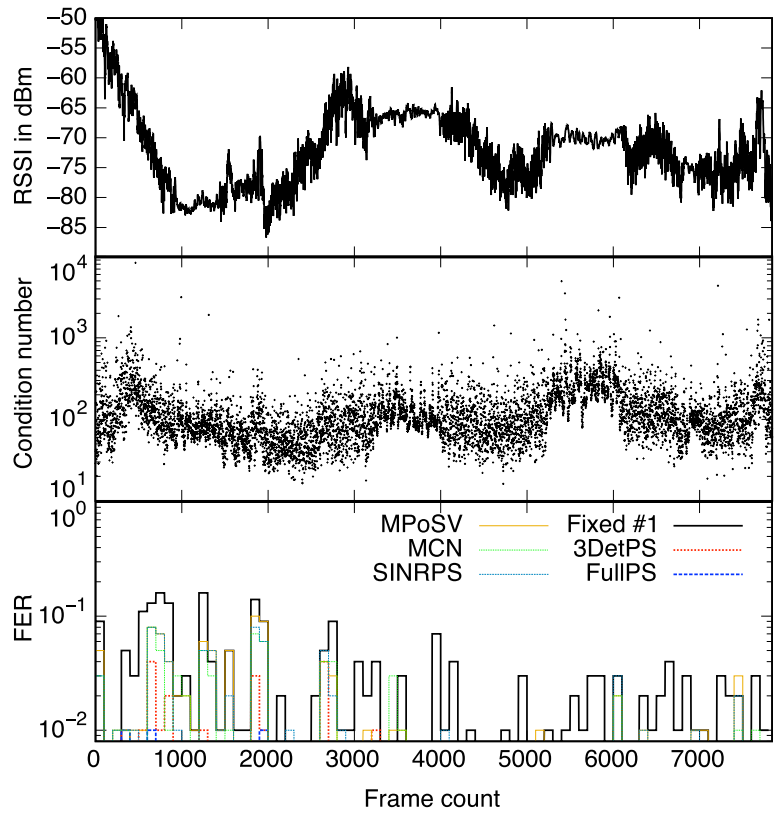

Fig. 13 RSSI, sum of condition numbers, and FER of trial 2. RSSI is averaged over six MSs.

and $\mathrm{MCN}$, these numbers are depending on whether an MS in charge of detection is in the selected MSs $\mathcal{L}^{*}$ or not. This number of SINRPS is stochastic, and was around 4.37 in average. The proposed 3Det and 3DetPS require the smallest number of waveforms, though a detected bit sequence gathering process is needed. The channel matrices based MS selection schemes require TSs (or CSI) of all the MSs to perform the selection, and issue control signaling to let MSs know the result. These are shown as additional traffic
Table 3 Number of transmitted waveforms in collaboration links.

\begin{tabular}{l|c|c|c}
\hline Scheme & Det. MS $\in \mathcal{L}^{*}$ & Det. MS $\notin \mathcal{L}^{*}$ & Additional traffic \\
\hline Fixed & 3 & 4 & \\
MPoSV & 3 & 4 & TS \& control \\
MCN & 3 & 4 & TS \& control \\
SINRPS & \multicolumn{2}{|c|}{$3-5$, average 4.37 } & TS \& control \\
\cline { 2 - 3 } 3Det & 3 & Bit sequences \\
3DetPS & 3 & Bit sequences \\
Full & \multicolumn{2}{|c|}{3} & \\
FullPS & 5 &
\end{tabular}

in Table 3.

In our implementation [21], 8 bits signed integer representation of the I and Q components was used for the received waveform exchange in the pulse-coded modulation mode, which means a total of 16 bits for representation of each symbol sample. Ignoring small fractions of the payload such as $\beta_{\mathcal{L}, m}, 4$ bits per symbol are sufficient for detected bit sequences exchange. Therefore, 3Det and 3 DetPS require $16 \times 3+4 \times 2=56$ bits/symbol traffic over the collaboration links, whereas Full and FullPS require $16 \times 5=80 \mathrm{bits} / \mathrm{symbol}$ traffic. Thus, $30 \%$ traffic reduction can be acheived.

\section{Conclusion}

This paper has presented new MS collaboration schemes that combine detected bit sequences obtained at multiple MSs. The performance of these schemes is shown not only by computer simulations but also by a measurement campaign. These schemes outperformed adaptive MS selection schemes based on MIMO channel matrices at the expense of additional signal processing complexity.

The proposed schemes employed the residual error coefficients as the metric for selection combining. This metric is an inherent value in the FD iterative equalization and thus, requires no extra computation nor redundancy. Detailed comparison with error-detection schemes is left for further study. The performance of the proposed schemes will be improved by selecting an appropriate set of helper MSs adaptively. This also requires further investigation.

\section{References}

[1] R. Dabora and S.D Servetto, "Broadcast channels with cooperating decoders,” IEEE Trans. Inf. Theory., vol.52, no.12, pp.5438-5454, Dec. 2006.

[2] T. Okubo and M. Itami, "A study on cooperative reception of one segment ISDB-T," Proc. 2008 International Symposium on Information Theory and its Applications, Auckland, Dec. 2008.

[3] M. Dohler, J. Dominguez, and H. Aghvami, "Link capacity analysis for virtual antenna arrays," Proc. IEEE 56th Vehicular Technology Conference, vol.1, pp.440-443, Vancouver, BC, Canada, Sept. 2002.

[4] H. Murata, "Collaborative interference cancellation for multiuser MIMO communication systems," IEICE Technical Report, RCS2013-201, Nov. 2013 (in Japanese).

[5] A. Maaref, J. Ma, M. Salem, H. Baligh, and K. Zarin, "Device-centric radio access virtualization for 5G networks," Proc. GLOBECOM 2014 Workshop, pp.887-893, Austin, Texas, Dec. 2014.

[6] Y. Kishiyama, "Initial thought on Beyond 5G radio access technologies," IEICE Society Conference, BS-2-2, p.S-11, Sept. 2017. 
[7] H. Murata and R. Shinohara, "Performance improvement of ZFprecoded MU-MIMO transmission by collaborative interference cancellation," IEICE Commun. Express, vol.4, no.5, pp.155-160, May 2015.

[8] F. Du and H. Murata, "Performance study of terminal collaborated MIMO reception experimental testbed in actual environment," Proc. 16th IEEE Asia Pacific Wireless Communications Symposium (APWCS2019), Singapore, Aug. 2019.

[9] H. Murata, R. Shinohara, and Y. Fujimoto, "Performance of adaptive mobile terminal selection schemes for collaborative MMSE linear MIMO detection," IEICE Commun. Express, vol.8, no.12, pp.662667, Dec. 2019.

[10] M. Arai and H. Murata, "Study of adaptive terminal selection schemes for terminal collaborated MIMO reception and its performance evaluation with actual received signals," IEICE Trans. Commun. (Japanese Edition), vol.J103-B, no.2, pp.110-118, Feb. 2020.

[11] F. Du and H. Murata, "Robustness of statistical antenna selection for collaborated MIMO reception: A feasibility study,” IEICE Commun. Express, vol.9, no.7, pp.324-329, July 2020.

[12] M. Kasai and H. Murata, "Performance comparison of adaptive terminal selection schemes for terminal collaborated MIMO reception using actual received signals," Proc. 2020 IEEE 91st Vehicular Technology Conference (VTC2020-Spring), Virtual Conference, May 2020

[13] F. Du, H. Murata, T. Nakahira, K. Ishihara, and T. Moriyama, "Study of estimated SINR based terminal selection in terminal collaborated MIMO reception," Proc. Genearl Conf. of IEICE, B-5-90, p.378, March 2020.

[14] T. Koike, H. Murata, and S. Yoshida, "Frequency-domain SC/MMSE iterative equalizer with MF approximation in LDPC-coded MIMO transmissions," IEEE 15th International Symposium on Personal, Indoor and Mobile Radio Communications, vol.24, pp.1414-1428, Sept. 2004.

[15] S. Minami, H. Nagano, and H. Murata, "Performance evaluation of iterative frequency domain equalization with LDPC code for user collaborated reception,” IEICE Technical Report, RCS2015-383, March 2016 (in Japanese).

[16] K. Kansanen and T. Matsumoto, "An analytical method for MMSE MIMO turbo equalizer EXIT chart computation," IEEE Trans. Wireless Commun., vol.6, no.1, pp.59-63, Jan. 2007

[17] M. Kasai, H. Murata, T. Nakahira, K. Ishihara, and T. Moriyama, "Effect of early stopping on error performance of iterative MIMO equalization: An experience in reality," IEICE Commun. Express, vol.9, no.10, pp.489-494, Oct. 2020.

[18] M. Kasai and H. Murata, "Study of terminal selection scheme using reliability of replica in terminal collaborated MIMO reception," Proc. URSI GA 2020, C14-03, Roma, Italy, Aug.-Sept. 2020.

[19] K. Mitsuyama, T. Ikeda, and T. Ohtsuki, "MIMO receiver with antenna subset selection: Algorithm and hardware implementation," IEICE Trans. Commun., vol.E96-B, no.4, pp.1039-1050, April 2013.

[20] S. Verdu, Multiuser Detection, Cambridge University Press, Cambridge, United Kingdom, 1998

[21] T. Chiba and H. Murata, "Experimental study of user collaborated reception using PCM and AF inter-terminal communications exploiting higher-frequency bands," IEICE Technical Report, RCS2017-390, March 2018 (in Japanese).

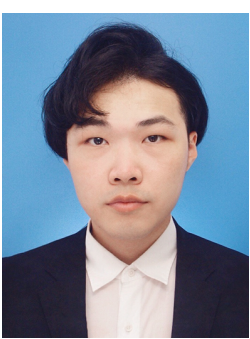

Fengning Du received his B.E. degree in Electrical and Electronic Engineering from Jilin University, China, in 2017, and his M.E. degree in Informatics from Kyoto University, Kyoto, Japan, in 2020. In 2020, he joined Huawei Technologies Co., Ltd., Research \& Development Group, China. His current research interest includes the areas of wireless LAN and IoT.

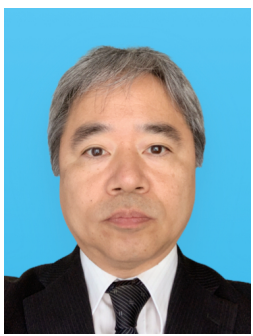

Hidekazu Murata received B.E., M.E., and Ph.D. degrees in electronic engineering from Kyoto University, Kyoto, Japan, in 1991, 1993, and 2000, respectively. In 1993, he joined the Faculty of Engineering at Kyoto University. From 2002 to 2006, he served as an Associate Professor at Tokyo Institute of Technology. He has been at Kyoto University since October 2006 and is currently an Associate Professor in the Department of Communications and Computer Engineering, Graduate School of Informatics. His major research interests include signal processing and its hardware implementation, particularly for application to cooperative wireless networks. Prof. Murata received the Young Researcher's Award from the IEICE of Japan in 1997, Ericsson Young Scientist Award in 2000, and Young Scientists' Prize of the Commendation for Science and Technology by the Minister of Education, Culture, Sports, Science and Technology in 2006; further, he was the recipient of the Best Paper Award of the IEICE in 2011 and 2013 as well as the IEEE ICC Best Paper Award in 2014. He is a member of the IEEE and ITE.

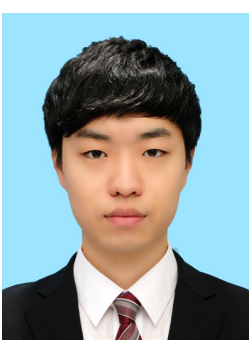

Mampei Kasai received his B.E. degree from Kyoto Institute of Technology, Kyoto, Japan, in 2019. He is currently studying towards his M.E. degree at the Graduate School of Informatics, Kyoto University. His research interests includes cooperative wireless networks.

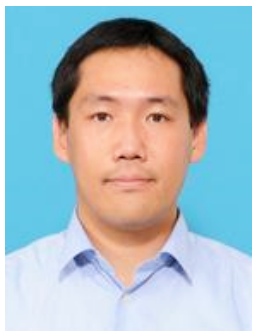

Toshiro Nakahira received his bachelor degree of maritime safety in Japan Coast Guard Academy in 2009 and his M.E. degree in informatics from Kyoto University in 2012. In 2012, he joined NTT Network Innovation Laboratories, Yokosuka, Japan. He is now working for NTT Access Network Service Systems Laboratories. He received the best research award of the fourth basic course workshop of IEICE Communication Quality (CQ) in 2017 and young engineer award from IEICE in 2019. He is a member

of IEICE. 


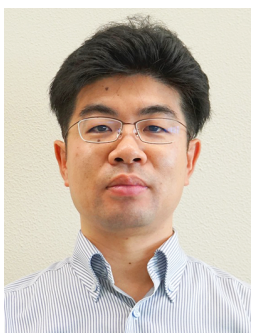

Koichi Ishihara received his B.E., M.E. and $\mathrm{Ph} . \mathrm{D}$. degrees in communications engineering from Tohoku University, Japan, in 2004, 2006 and 2011, respectively. He joined NTT in 2006. $\mathrm{He}$ is currently a Senior Research Engineer in the NTT Access service systems laboratories where he is researching and developing a high efficiency wireless access systems. He received the Young Engineer Award in 2009, the RCS (Radio Communication Systems) Active Research Award in 2011, the Achievement Award in 2014, and the Best Tutorial Paper Award in 2014 from IEICE. He is a Member of IEEE and IEICE.

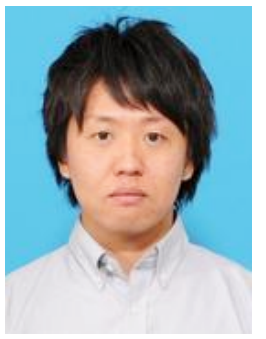

Motoharu Sasaki received his B.E. degree in engineering and his M.E. and Ph.D. degrees in information science and electrical engineering from Kyushu University, Fukuoka, Japan in 2007, 2009, and 2015, respectively. In 2009, he joined NTT Access Network Service Systems Laboratories, Yokosuka, Japan. He has been engaged in research on propagation modeling for various wireless communication systems; propagation modeling of interference between mobile terminals for spectrum sharing wireless access systems, propagation modeling in VHF bands for emergency wireless systems, and propagation modeling in high frequency bands for Fifthgeneration mobile wireless communication systems. From 2018, he is currently a Research Engineer in Planning Section of NTT Access Network Service Systems Laboratories. He received the Young Researcher's Award and the Best Paper Award from the Institute of Electronics, Information and Communication Engineers (IEICE) in 2013 and 2014, respectively. He received the Best Paper Award in International Symposium on Antennas and Propagation (ISAP) in 2016. He received Young Engineer Award from IEEE AP-S Japan chapter in 2016. He also received the Young Researcher Award and the Excellent Paper Award from IEICE Technical Committee on Antennas and Propagation in 2012 and 2018, respectively. He is a member of IEEE.

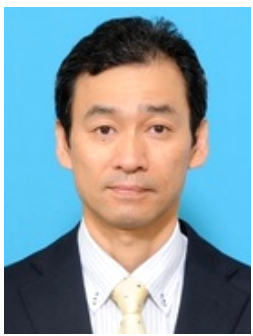

Takatsune Moriyama received his B.E. and M.E. degrees from Muroran Institute of Technology, Japan, in 1991 and 1993. He joined NTT in 1993. Since 1999, he worked at NTT Communications, where he was in charge of network service development and operation for corporate customers. He has been in his current position since July 2019. 